\title{
Fluoxetin-induced pulmonary granulomatosis
}

\author{
E. de Kerviler*, J. Trédaniel**, G. Revlon*, O. Groussard+, G. Zalcman**, \\ J-M. Ortoli**, M. Espié ${ }^{++}$A. Hirsch**, J. Frija*
}

Fluoxetin-induced pulmonary granulomatosis. E. de Kerviler, J. Trédaniel, G. Revlon, O. Groussard, G. Zalcman, J-M. Ortoli, M. Espié, A. Hirsch, J. Frija. @ERS Journals Ltd 1996.

ABSTRACT: A patient treated with fluoxetin for a manic depressive disorder developed pulmonary inflammatory nodules with noncaseating giant cell granulomas, interstitial pneumonia and non-necrotizing vasculitis, whilst remaining asymptomatic.

A progressive resolution of pulmonary nodules occurred after withdrawal of the offending agent, and the chest radiograph returned to normal in 9 months. The diagnosis was assessed by an open lung biopsy.

Eur Respir J., 1996, 9, 615-617.

\begin{abstract}
*Service de Radiologie, **Service de Pneumologie and ${ }^{++}$Service de Cancérologie, Hôpital Saint-Louis, Paris, France. + ${ }^{+}$Service d'Anatomie et Cytologie Pathologique Hôpital Beaujon, Clichy, France.

Correspondence: E. de Kerviler, Service de Radiologie, Hôpital Saint-Louis, 1 avenue Claude-Vellefaux, 75010 Paris, France

Keywords: Fluoxetine, lung - effect of drugs, lung - granuloma, lung - nodules Received: August 91995

Accepted after revision October 91995
\end{abstract}

The recognition of drug-induced pneumonia has become an increasingly important area in pulmonary disorders. The common imaging features are either interstitial diffuse pneumonia or pulmonary oedema [1-3]. Fluoxetin is an antidepressant, neither tricyclic nor monoamine oxidase inhibitor (MAOI), inhibiting the reuptake of serotonin. We report the case of a patient treated with fluoxetin, who developed multiple round pulmonary nodules simulating metastases.

\section{Case report}

A 49 year old woman was referred to an outside hospital in October 1992 for the surgical treatment of an

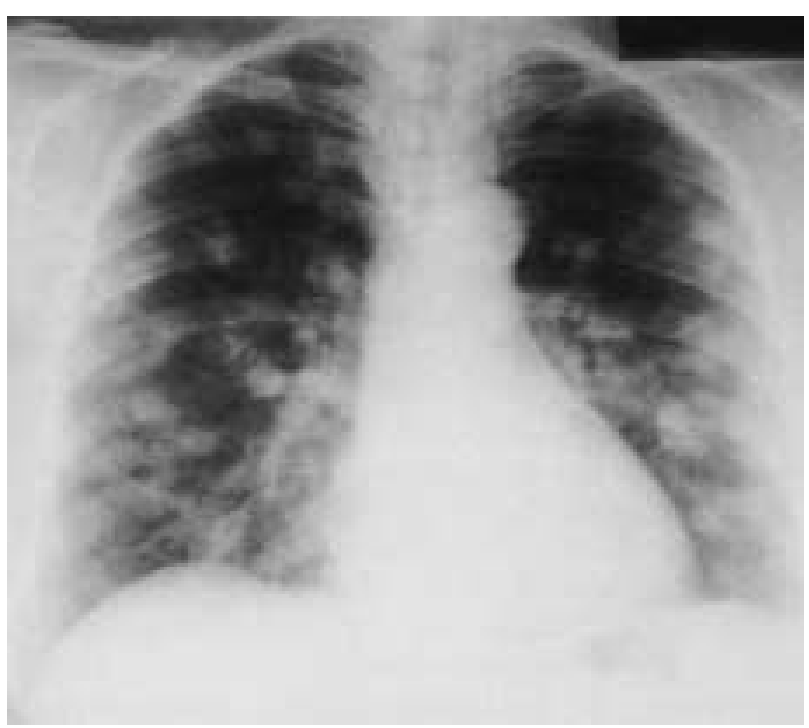

Fig. 1. - Chest plain radiograph shows multiple bilateral round nodules simulating a metastatic disease. anterior abdominal wall hernia. The preoperative chest radiograph displayed multiple round bilateral pulmonary nodules (fig. 1). A previous chest radiograph performed in February 1992 was normal. The patient had been treated for 10 years with lithium for manic depressive disorder. A treatment with Fluoxetin (20 mg daily) was given from June to September 1992. Rigorous questioning did not detect any exposure to inhaled antigens or toxic gases and aerosols.

At the time of admission, the patient was asymptomatic and her general status was good. Breath sounds were normal. A chest computed tomography (CT) scan showed well-defined pulmonary nodules with a diameter of 5-20 mm (fig. 2). The lesions were bilateral, and predominated at the periphery of the lungs. Some nodules were in contact with the pleura, others were connected to vessels. Neither mediastinal lymphadenopathy nor pleural involvement was found. Since the imaging

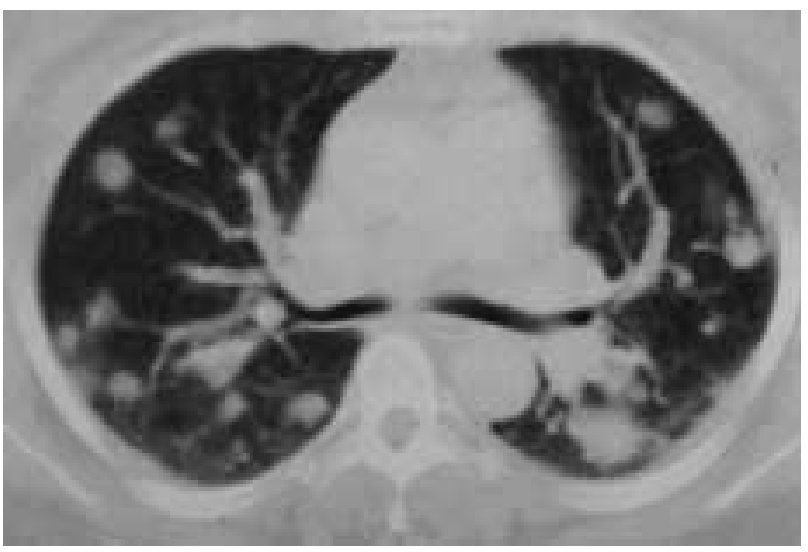

Fig. 2. - Chest computed tomography scan shows bilateral round nodules with well-defined margins. The nodules predominate at the periphery of the lungs. Some are connected to vessels. 


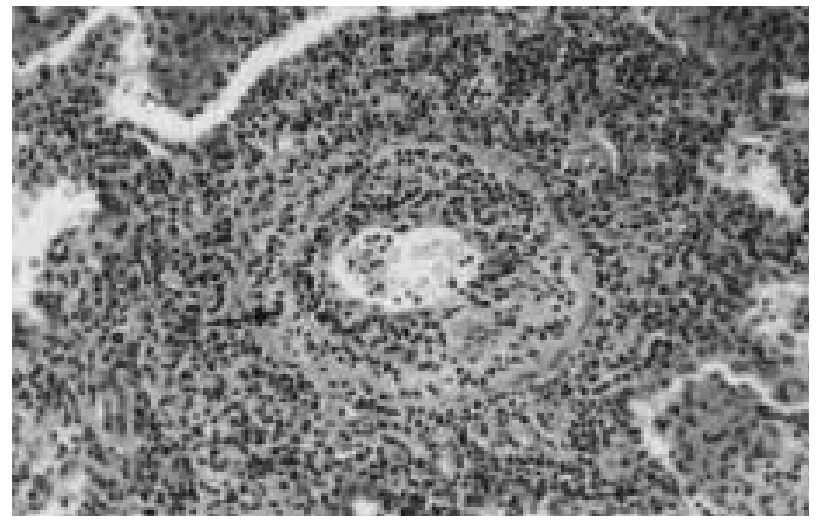

Fig. 3. - Lymphocytic angiitis. The wall of a venule (arrow) is infiltrated by lymphoid and plasma cells. (Haematoxylin and eosin stain; original magnification $\times 250$ ).

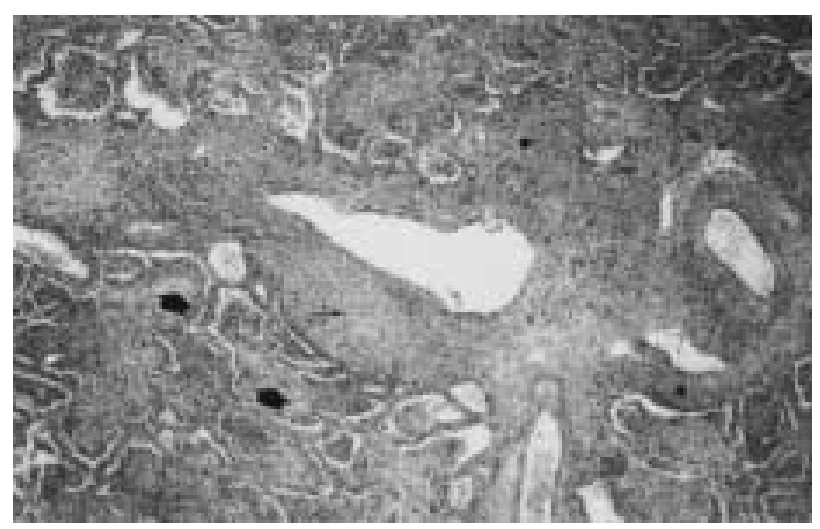

Fig. 4. - Interstitial and organizing pneumonia. Thickening of alveolar wall by granulomatous infiltrates (asterisks) and the endoluminal buds (large arrow) of connective tissue. At the centre of the picture, granuloma infiltrates the wall of a vein (small arrow). (Haematoxylin and eosin stain; original magnification $\times 100$ ).

feature was suggestive of metastatic lesions, multiple investigations were initiated to detect a primary tumour and/or other metastases. However, all remained negative. Blood cell count was normal. No malignant cells were found in biopsies obtained by fibreoptic bronchoscopy. An open lung biopsy showed inflammatory nodules with noncaseating giant cell granulomas, interstitial and organizing pneumonia and polyclonal B- and T-lymphocytic angiitis, without necrosis of the vessels walls (figs. 3 and 4). Bronchoalveolar lavage performed at that time showed a lymphocytosis corresponding to $40 \%\left(160 \times 10^{3}\right.$ cells. $\mathrm{mL}^{-1}$ ) of recovered cells. Diffusing capacity for carbon monoxide was mildly reduced. The remaining pulmonary functions were normal. Arterial blood gas analysis, breathing room air, was normal.

A progressive resolution of pulmonary nodules occurred and the chest radiograph returned to normal in 9 months. Resumption of drug therapy was not attempted. The patient is still well without respiratory illness 30 months later.

\section{Discussion}

The list of drugs implicated in lung injury has grown rapidly during recent decades. In a review of the subject published in 1992, RosENOw et al. [1] reported 60 therapeutic agents associated with pulmonary parenchymal damage. As a comparison, the same authors reported only 19 drugs responsible for pulmonary damage in 1972 [4]. More recently, Aкоun et al. [5] listed 111 therapeutic agents which have been implicated in pulmonary diseases. Even though not uncommon, the diagnosis of drug-induced disease may be difficult because the clinical and biological symptoms are often nonspecific, the patient's underlying disease may be responsible for pulmonary abnormalities and the use of multiple drugs reduces the chances of identifying the offending agent.

Fluoxetin is a new antidepressant, neither tricyclic nor monoamine oxidase inhibitor (MAOI), inhibiting the reuptake of serotonin. Fluoxetin-induced pulmonary damage has previously been reported [6]; a patient developed an interstitial lung disease whilst taking fluoxetine. A chest radiograph showed "a diffuse interstitial shadow-ing throughout both lung fields". To our knowledge, we report here the first case of granulomatous reaction in the lung occurring in a patient treated by fluoxetin [7]. In our opinion, the drug was responsible for the pulmonary disease for the following reports. Firstly, the underlying psychiatric disease is not likely to have induced pulmonary lesions, and no other systemic disease could be detected. Secondly, no therapy except fluoxetin had been introduced in the past 2 yrs. Finally, the withdrawal of fluoxetin without specific treatment led to a favourable outcome. The only manifestation of the disease was the presence of pulmonary nodules found on chest radiograph and CT. Such a pattern is usually seen in cases of lung metastases, but also in infectious processes, such as histoplasmosis and coccidiomycosis as well as immunological disorders, such as Wegener granulomatosis and rheumatoid arthritis. The imaging feature was very different from that currently observed in hypersensitivity pneumonitis, which consists mainly in interstitial diffuse pneumonia and pulmonary oedema [13].

The diagnosis of drug-induced granulomatosis and angiitis was based on the temporal relationship between fluoxetine and lung disease, and on histopathological findings. Granulomatous angiitis is a form of vasculitis characterized by a marked infiltration of arteries and veins with lymphocytes, plasma cells and macrophages, and the presence of noncaseating granulomas [8]. The major drugs involved are penicillin, ampicillin and sulphonamides [8]. The two main differential diagnoses at histopathology were lymphomatoid granulomatosis and sarcoidosis. The first hypothesis was ruled out because the infiltrate was polyclonal, made of B- and T-lymphocytes with a round regular nucleus. Moreover, the immunohistochemical analysis demonstrated expression of both kappa and lamba light chains. The second hypothesis was also eliminated because the histological pattern was not that of sarcoid granulomas.

In summary, this patient presented with a fluoxetininduced pulmonary granulomatosis simulating metastatic lesions. All the biological investigations remained negative. The diagnosis was assessed by open lung biopsy. 


\section{References}

1. Rosenow III E, Myers J, Swensen S, Pisani R. Druginduced pulmonary disease: an update. Chest 1992; 102: 239-250.

2. Cooper J, White D, Matthay R. Drug-induced pulmonary disease. Part 2. Noncytotoxic drugs. Am Rev Respir Dis 1986; 133: 488-505.

3. Gregory S, Grippi M. The clinical diagnosis of druginduced pulmonary disorders. J Thorac Imag 1991; 6: $8-18$.
4. Rosenow III E. The spectrum of drug-induced pulmonary disease. Ann Intern Med 1972; 77: 977-991.

5. Akoun G, Milleron B, Cadranel J, Mayaud C. Pneumopathies médicamenteuses. In: Godard P, Bousquet J, Mayaud C, eds. Maladies Respiratoires. Masson, 1993; 791-797.

6. Bass S, Colebatch H. Fluoxetine-induced lung damage. Med J Aust 1992; 156: 364-365.

7. Wernicke J. The side effect profile and safety of fluoxetine. J Clin Psychiatry 1985; 46: 59-67.

8. Pietra G. Pathologic mechanisms of drug-induced lung disorders. J Thorac Imag 1991; 6: 1-7. 